\title{
Caracterización microbiológica de polen seco y congelado en Viracachá-Colombia
}

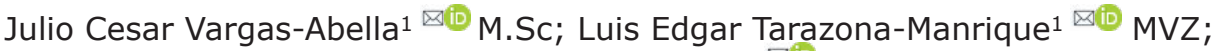 \\ Roy José Andrade-Becerra1* ${ }^{1 *}$ Ph.D.
}

${ }^{1}$ Universidad Pedagógica Y Tecnológica De Colombia. Facultad de ciencias agropecuarias. Escuela de medicina veterinaria y zootecnia. Laboratorio de microbiología veterinaria. Grupo de investigación GIPATRACOL. Tunja, Colombia.

*Correspondencia: roy.andrade@uptc.edu.co

Recibido: Noviembre 2019; Aceptado: Mayo 2020; Publicado: Agosto 2020.

\section{RESUMEN}

Objetivo. Caracterizar microbiológicamente el polen seco y congelado producido en el municipio de Viracachá-Boyacá. Materiales y métodos. A través de un estudio transversal descriptivo cuantitativo se tomaron muestras de 5 apiarios, cada uno con 10 colmenas, separando el polen en seco y congelado, determinando para cada muestra: aerobios mesófilos, coliformes totales, coliformes fecales, Staphylococcus aureus, Clostridium sulfito reductor y hongos. Los datos obtenidos se analizaron de acuerdo a normatividades internacionales y se compararon con resultados de investigaciones en otros países. Resultados. Se encontraron coliformes totales y fecales en tres de los cinco apiarios evaluados y solo en muestras de polen seco. Además, en dos apiarios cuando se analizó polen seco se encontró Staphylococcus aureus. Los resultados microbiológicos de la mayoría de las muestras se encuentran dentro de los rangos de algunas normatividades internacionales, sin embargo, los mejores resultados en cuanto a calidad microbiológica se determinaron para el polen congelado. Conclusiones. El proceso de congelamiento del polen ofrece ventajas relativas al mantenimiento de la calidad microbiológica en comparación con el proceso de secado. Se hace necesario evaluar la calidad microbiológica de ambos productos a través del tiempo de almacenamiento.

Palabras clave: Producto apícola; Apis mellifera; almacenamiento; microbiología; polen; calidad sanitaria (Fuente: $C A B, D e C S$ ).

\section{ABSTRACT}

Objective. Microbiologically characterize dry and frozen pollen produced in the municipality of Viracachá-Boyacá. Materials and methods. Through a quantitative descriptive cross-sectional study, samples from 5 apiaries were taken, each with 10 hives, separating the pollen dry and frozen, determining for each sample: mesophilic aerobes, total coliforms, fecal coliforms, Staphylococcus aureus, Clostridium sulfite reducer, and fungus. The data obtained were analyzed according to international regulations and compared with research results in other countries. Results. Total and fecal coliforms were found in three of the five apiaries evaluated and only in dried pollen samples. Also, in two apiaries when dry pollen was analyzed, Staphylococcus aureus was found. The microbiological

Como citar (Vancouver).

Vargas-Abella JC, Tarazona-Manrique LE, Andrade-Becerra RJ. Caracterización microbiológica de polen seco y congelado en Viracachá-Colombia. Rev MVZ Córdoba. 2020; 25(3):e1854. https://doi.org/10.21897/rmvz.1854 
results of most samples are within the ranges of some international regulations; however, the best results in terms of microbiological quality were determined for frozen pollen. Conclusions. The pollen freezing process offers advantages related to maintaining microbiological quality compared to the drying process. It is necessary to evaluate the microbiological quality of both products throughout the storage time.

Keywords: Bee product; Apis mellifera; storage; bee pollen; microbiology; sanitary quality (Sources: $C A B, D e C S)$.

\section{INTRODUCCIÓN}

La apicultura es un renglón económico de gran importancia en diversos países debido a que genera gran variedad de productos que permiten a los productores obtener diversos niveles de ingresos por la venta de todos ellos aprovechando las tendencias de consumo con productos naturales $(1,2)$ Los productos apícolas se pueden clasificar según su origen en: productos colectados sin transformación (polen y propóleos), colectados con transformación (miel y frutimiel), derivados de secreción (cera, jalea real, apitoxina) y otros como (reinas, núcleos, paquetes, larvas de zánganos, servicios de polinización) (3).

El polen apícola, deriva de la aglutinación de diversos granos de polen que las abejas recolectan de las flores, sumado a secreciones salivares y pequeñas cantidades de néctar o miel $(4,5,6)$. Este producto es usado tradicionalmente en la alimentación humana y se ha demostrado que tiene propiedades antimicrobianas, antiinflamatorias, antitumorales, antimutagénicas, antioxidantes, antialérgicas, antivirales, hipolipidémicas, hipoglicémicas, hepato y renoprotectivas e inmunoestimulantes $(7,8,9,10,11)$

Debido a su manipulación durante el proceso de recolección por parte de las abejas, de su colección en la colmena, transporte y almacenamiento, el polen está expuesto a diversas fuentes de contaminación que podrían alterar sus características organolépticas y, dependiendo el tipo de microorganismo que llegue a crecer en este, poner en riesgo su calidad sanitaria y la transmisión de enfermedades ya sea por el consumo del microorganismo per se, o por el consumo de sustancias tóxicas producto de su metabolismo intermedio, como el caso de los hongos $(12,13,14,15)$.

En Colombia, los estudios relacionados con la cadena apícola son escasos en comparación con otros tópicos pecuarios, esto genera un vacío de información científica de calidad que permita el desarrollo de políticas públicas encaminadas al mejoramiento de la cadena productiva. Esto genera que, entre otras cosas, no exista en el país una normatividad con respecto a la calidad microbiológica de este tipo de productos, y debido a que en su mayoría se consumen de forma directa podrían representar una fuente propicia para la generación o transmisión de enfermedades, por tanto, su monitoreo microbiológico debe ser riguroso para ofrecer productos en óptimas condiciones $(4,16)$.

Por ello, el objetivo del presente trabajo fue caracterizar microbiológicamente el polen seco y congelado que se produce en el municipio de Viracachá- Boyacá.

\section{MATERIALES Y MÉTODOS}

Tipo y sitio de estudio. Se realizó un estudio de corte transversal, descriptivo cuantitativo. El estudio se realizó en cinco apiarios ubicados en el municipio de Viracachá- Boyacá, este se encuentra ubicado en la provincia de Márquez, región centro oriente del departamento de Boyacá, con una altura sobre el nivel del mar de 2500 metros, temperatura promedio de $15^{\circ} \mathrm{C}$ y precipitación media anual de 824 mm (17).

Muestreo. Se seleccionaron cinco apiarios, con 10 colmenas cada uno, con trampa caza polen tipo canadiense. Cada apiario está ubicado en una vereda diferente del municipio de Viracachá, las veredas tienen una diferencia de altura de 200 metros. El muestreo se realizó en cada una de ellas, una sola vez semanalmente, durante los meses de agosto-septiembre de 2019.

El polen de los cajones recolectores de cada apiario fue envasado en recipientes plásticos independientes y transportados hasta un sitio de almacenamiento ubicado en la vereda Pueblo viejo. Allí, el polen de cada apiario fue sometido a dos limpiezas, la primera con aire (tipo mecánico) $y$, en seguida, una limpieza manual, con ayuda de lupa, para la extracción de elementos externos al 
polen. Posteriormente cada muestra se separó, un $50 \%$ fue llevado a máquina de secado a una temperatura entre $45-50^{\circ} \mathrm{C}$ durante 8 horas, mientras que el restante fue llevado a congelación a una temperatura de $0^{\circ} \mathrm{C}$ durante 8 horas.

Se tomaron 200 gramos de muestra de cada apiario y se envasaron en recipientes de vidrio de tapa rosca estériles, es decir, 100 gramos de polen congelado y 100 gramos de polen seco, y fueron procesados al día siguiente en el laboratorio de microbiología veterinaria de la UPTC.

Transporte. Las muestras fueron transportadas el mismo día de su recolección. El polen seco se transportó en estibas plásticas a temperatura ambiente. Por su parte, el polen congelado fue transportado en cavas con hielo en su interior, monitorizando la temperatura para que esta oscilara entre 0 y $4^{\circ} \mathrm{C}$.

Al momento del arribo al laboratorio de microbiología veterinaria las muestras de polen congelado se mantuvieron a temperatura de $0^{\circ} \mathrm{C}$ hasta el día siguiente para su procesamiento. Las muestras de polen seco, se mantuvieron a temperatura ambiente durante el mismo tiempo.

Análisis microbiológico. Las muestras fueron abiertas en una cabina de flujo laminar y allí, 25 gramos de cada muestra fueron diluidos en $225 \mathrm{~mL}$ de solución salina peptonada estéril y fueron homogeneizadas en un homogeneizador orbitario a $200 \mathrm{rpm}$ durante 10 minutos. Posteriormente, se realizaron diluciones en 9 $\mathrm{mL}$ de solución peptonada estéril. Para ello, se tomó $1 \mathrm{~mL}$ de la primera dilución y se añadió en el tubo para completar $10 \mathrm{~mL}$, luego se realizó el mismo procedimiento hasta completar tres diluciones. Las colonias formadas en las placas fueron contadas y expresadas como unidades formadoras de colonia/gramo (UFC/gr) $(5,18,19)$.
Los análisis microbiológicos fueron realizados con $1 \mathrm{~mL}$ de la última solución, los microorganismos a determinar fueron: Aerobios mesófilos (placas petrifilm incubadas a $30^{\circ} \mathrm{C}$ durante 3 días), coliformes totales (agar violeta rojo bilis glucosaVRBG, estas fueron incubadas a $35^{\circ} \mathrm{C}$ y colonias de color negro-rojizas de $0.5 \mathrm{~mm}$ o más en diámetro fueron contadas 24 horas después de la siembra), coliformes fecales (placa petrifilm ${ }^{\circledR}$ para coliformes, incubadas a una temperatura de $30^{\circ} \mathrm{C}$ durante 3 días), Staphylococcus aureus (agar Baird-Parker e incubados a $37^{\circ} \mathrm{C}$ durante 48 horas), Esporas Clostridium sulfito reductor (se colocó la dilución en baño de agua a $80^{\circ} \mathrm{C}$ por 10 minutos y luego fue inoculado en cajas de petri que contenían agar Sulfito-Hierro, fueron incubadas durante 2 días a $37^{\circ} \mathrm{C}$ bajo condiciones anaeróbicas), mohos y levaduras (agar Saboureaud, y fueron incubadas durante 5 días a $\left.30^{\circ} \mathrm{C}\right)(5,18)$.

Para descartar que la solución peptonada alterase los resultados, $1 \mathrm{ml}$ de esta fue inoculada en cada uno de los agares anteriormente mencionados y los resultados se muestran en la tabla 1.

Análisis estadístico. Se realizó un análisis de varianza (ANDEVA) para los valores de crecimiento de cada uno de los microorganismos evaluados para cada tipo de polen (seco y congelado) entre cada apiario tomando como referencia la mínima diferencia significativa del procedimiento de Fisher, con un nivel de confianza del 95\% con el software StatgraphicsCenturion ${ }^{\circledR}$ versión Windows 10, los valores significativamente diferentes son expresados en $(p<0.05)$.

Tabla 1. Resultados de los análisis microbiológicos efectuados al polen seco y congelado.

\begin{tabular}{|c|c|c|c|c|c|c|c|c|c|c|c|}
\hline \multirow{2}{*}{ Análisis microbiológico* } & \multicolumn{2}{|c|}{ Apiario 1} & \multicolumn{2}{|c|}{ Apiario 2} & \multicolumn{2}{|c|}{ Apiario 3} & \multicolumn{2}{|c|}{ Apiario 4} & \multicolumn{2}{|c|}{ Apiario 5} & \multirow{2}{*}{$\begin{array}{c}\text { Control } \\
\text { NA }\end{array}$} \\
\hline & P.S. ${ }^{1}$ & P.C. ${ }^{2}$ & P.S. & P.C. & P.S. & P.C & P.S. & P.C. & P.S. & P.C. & \\
\hline Aerobios mesófilos $\times 10^{3}$ & 12 & 4 & 10 & 6 & 11 & 3 & 9 & 2 & 12 & 6 & 0 \\
\hline Coliformes totales $\times 10^{3}$ & 2 & 0 & 0 & 0 & 3 & 0 & 1 & 0 & 0 & 0 & 0 \\
\hline Coliformes fecales $\times 10^{3}$ & 1 & 0 & 0 & 0 & 2 & 0 & 1 & 0 & 0 & 0 & 0 \\
\hline Staphylococcus aureus $\times 10^{3}$ & 2 & 0 & 1 & 0 & 0 & 0 & 0 & 0 & 0 & 0 & 0 \\
\hline Clostridium s. reductor $\times 10^{3}$ & 0 & 0 & 0 & 0 & 0 & 0 & 0 & 0 & 0 & 0 & 0 \\
\hline Mohos y levaduras $\times 10^{3}$ & 2 & 0 & 5 & 2 & 4 & 3 & 6 & 0 & 1 & 0 & 0 \\
\hline
\end{tabular}

*Expresado en UFC/gr. ${ }^{1}$ P.S: Polen Seco. ${ }^{2}$ P.C: Polen Congelado. NA: No Aplica 


\section{RESULTADOS}

La tabla 1 muestra los resultados de los análisis microbiológicos efectuados a las muestras de polen y a la solución peptonada. Se evidencia que no existe crecimiento de ningún microorganismo desde la solución peptonada, por lo cual, los resultados muestran características microbiológicas del polen. El menor crecimiento de microorganismos se determinó en las muestras de polen congelado (Tabla 1 ).

La figura 1 muestra las diferencias estadísticas encontradas para los resultados de cada uno de los apiarios evaluados. Se evidencia que existen diferencias estadísticamente significativas entre el polen seco y congelado en aquellos que presentaron crecimiento de microorganismos. No se evidenció la presencia de esporas de Clostridium sulfito reductor en ninguna de las muestras de polen de todos los apiarios evaluados.

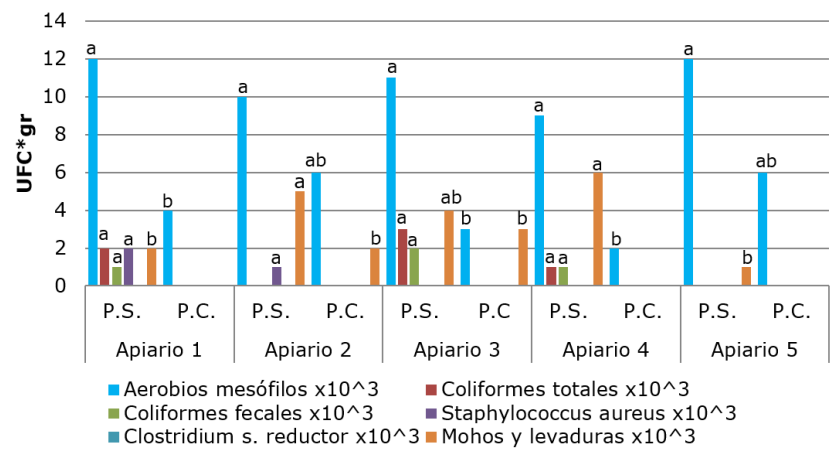

Figura 1. ANDEVA para los resultados de cada uno de los análisis microbiológicos. Las barras con la misma letra indican que pertenecen a un mismo grupo estadístico.

\section{DISCUSIÓN}

En Colombia no está, por parte de las autoridades nacionales, los rangos de calidad microbiológica en polen o demás productos apícolas que son destinados para consumo humano, esto conlleva a una gran problemática de orden sanitario debido a la posible intoxicación alimentaria derivada de los productos apícolas no solo por la presencia de microorganismos patógenos en el alimento, sino por la posible producción de toxinas producto de su metabolismo intermedio, tal y como sucede con algunos hongos $(2,14)$.

La contaminación microbiológica de los productos alimenticios puede suceder en diversas fases, debido al inadecuado manejo durante la recolección, transporte, procesamiento y embalaje (18). En el caso de los productos apícolas, estos pueden llegar a contaminarse desde el momento en el cual la abeja realiza la recolección y producción, situación influenciada principalmente por variables climáticas (2).

En un estudio en Colombia, García et al (20) determinaron los microorganismos que crecen en polen maduro de Apis mellifera (luego de su procesamiento a nivel de colmena) mostró crecimientos de microorganismos tales como: Streptococcus sp, Micrococus sp, Pseudomona $s p$, Yersinia $s p$ y Arthrobacter $s p$, asociándolos principalmente a los microorganismos presentes en el intestino de las abejas. Estos microorganismos no se determinaron en este estudio, sin embargo, muestra la correlación entre la presencia de microorganismos en el polen y el proceso mismo de producción del polen.

Los resultados muestran que existe una diferencia estadísticamente significativa entre los resultados para polen seco y congelado para resultados de aerobios mesófilos. En Argentina, la normatividad nacional fija algunos valores para los resultados microbiológicos de polen, para mesófilos aerobios el rango máximo fijado es de $150 \times 10^{3} \mathrm{UFC} / \mathrm{gr}$, lo cual indica que los dos tipos de polen evaluados en este estudio cumplen con esta norma, de la misma forma que con las normas de países como México, Brasil y la Unión Europea cuyos resultados deben ser menores a $1 \times 10^{4}$ UFC/gr $(1,21,22,23)$ Esto muestra, que aun cuando ambos procesos satisfacen estas normas internacionales, los mejores resultados microbiológicos los arrojó el polen congelado (Tabla 1).

El número de estos microorganismos refleja la calidad microbiológica de los productos apícolas, resultados elevados de estos revelan contaminación del material de las trampas, inadecuada limpieza y desinfección de las superficies de estas, insuficiente higiene en la preservación del polen, inadecuadas condiciones de almacenamiento con respecto a la temperatura o una combinación de algunas de estas circunstancias $(2,24)$.

Los coliformes totales y fecales se constituyen como uno de los microorganismos de mayor importancia epidemiológica debido a su capacidad de generar enfermedades de origen alimenticio, $y$, por tanto, no deberían estar presentes en muestras de alimentos destinados a consumo humano directo (1). La tabla 1 muestra el 
crecimiento de coliformes totales y fecales en tres de los cinco apiarios evaluados, y solo en muestras de polen seco.

Las normatividades de los países anteriormente mencionados exigen la ausencia de estos microorganismos en muestras de polen, por tanto, estos tres apiarios incumplen con estas normativas. Resulta relevante recalcar que, en ninguna muestra de polen congelado, existió crecimiento de coliformes, tanto fecales como totales $(1,21,22,23)$. Además, es necesario dejar claro que no se evaluó la presencia de estos microorganismos en el momento de la colecta, por ello, no es posible afirmar en qué momento la muestra pudo haberse contaminado.

Estudios en Portugal han reportado la ausencia de estos microorganismos en el total de muestras de polen comercial y polen orgánico tales como Estevinho et al (25), Nogueira et al (26) y Fea's et al (27), situación diferente a la reportada en el presente estudio. La contaminación con coliformes tanto fecales como totales, es un indicador de las condiciones higiénicas que se presentan en el material los insumos, equipos e instrumentos utilizados para la recolección y la manufactura (2).

Para el caso de Staphylococcus aureus, este no debe estar presente en el polen según las normatividades mexicana y de la Unión europea $(1,22)$, sin embargo, en dos apiarios cuando se analizó el polen seco, este microorganismo fue aislado; además, en estos mismos apiarios, en las muestras de polen congelado, no se evidenció crecimiento del agente (Tabla 1 ).

La normatividad cubana (28), determina la ausencia de colonias de Clostridium sulfito reductor debido a su potencial patogenicidad para el ser humano, todas las muestras de polen tanto seco como congelado muestran la ausencia del crecimiento de este microorganismo, con lo cual, estas muestras cumplirían con la norma mencionada.

De la misma manera, en un estudio en Brasil (2) determinó la ausencia de estos microorganismos en muestras de polen recolectadas durante un período de 3 años consecutivos en ocho distintos distritos de diversas partes del país.

La Unión Europea (1) permite hasta 50.000 UFC/ gr de mohos y levaduras en muestras de polen, por lo cual, bajo esta normatividad todas las muestras de polen cumplirían con lo estipulado, por otro lado, la normatividad argentina (21), brasilera (23) y cubana (28) admiten un máximo de $100 \mathrm{UFC/gr}$ de estos mismos microorganismos, por lo tanto, las muestras de polen no cumplirían con estas normatividades.

La presencia de estos microorganismos está asociada principalmente con las condiciones medioambientales en donde se encuentran ubicados los apiarios, y sirven como un parámetro para determinar condiciones adecuadas 0 inadecuadas del manejo de estos $(2,26)$.

En diversos países se ha analizado también la presencia de microorganismos contaminantes de polen, (18) en Argelia, determinó la presencia de mesófilos aerobios en muestras de polen seco de tres apiarios de distintos municipios encontrando valores de $620 \times 10^{3}, 1 \times 10^{3}$ y $470 \times 10^{3} \mathrm{UFC} / \mathrm{gr}$ los cuales son valores elevados comparados a los que se determinaron en este estudio para cualquiera de los apiarios evaluados. Así mismo, para el caso de los coliformes totales, encontraron valores de $300 \times 10^{3}, 01 \times 10^{3}$ y $19 \times 10^{3} \mathrm{UFC} / \mathrm{gr}$, resultados mayores a los determinados en este estudio, con la posibilidad, por ende, de que en Colombia las condiciones de manejo del producto sean mejores. Estos mismos autores cuando analizaron los mohos y las levaduras, sus valores fueron 400, 50 y $230 \mathrm{UFC} / \mathrm{g}$, resultados menores a los encontrados en este estudio para el polen seco o congelado en cualquier apiario.

En un estudio similar realizado en Brasil en ocho estados en donde se evaluó la calidad del polen deshidratado, los valores de mesófilos aerobios oscilaron entre $<10$ hasta $1.10 \times 10^{4} \mathrm{UFC} / \mathrm{g}$, los mohos y las levaduras oscilaron entre $<10$ hasta $7.67 \times 10^{3} \mathrm{UFC} / \mathrm{g}$, y los coliformes totales desde $<10$ hasta $2.80 \times 10^{3} \mathrm{UFC} / \mathrm{g}$, relacionando estos hallazgos con inadecuadas prácticas de higiene durante los pasos del proceso de manipulación, procesamiento y embalaje, incluso con la contaminación de las plantas o también, durante el momento en el cual el polen permanece en las trampas, debido a que está expuesto a diversos factores medioambientales, sumadas a ciertas temporadas que podrían favorecer condiciones para el crecimiento de microorganismos $(2,4)$.

Por otro lado, en una especie de abejas sin aguijón (Malipona mandacaia) Santa Bárbara et al (29) también en Brasil en las regiones de João Dourado y Uibaí, no encontraron crecimiento microbiológico de Salmonella $s p$, clostridios reductores de sulfito, coliformes fecales, Escherichia coli, Staphylococcus aureus, mohos 
y levaduras, contrastando ampliamente con los resultados obtenidos en este estudio, debido a que aquí, estos microorganismos, a excepción de Salmonella sp y Eschericha coli que no fueron evaluados, mostraron crecimiento en las muestras recolectadas. Los mesófilos aerobios, por otra parte, si fueron determinados en ese estudio, encontrando valores de $121.58 \mathrm{UFC} / \mathrm{g}$ para la región de João Dourado y de 375.67 UFC/g para la región de Uibaí, resultados que son menores a los determinados en este estudio para cualquiera de los dos municipios brasileros.

Las variaciones con respecto a este último grupo de microorganismos entre los estudios pudo deberse principalmente a las diferencias en la capacidad antimicrobiana que muestra el polen de cada especie de abejas, por ejemplo, Santa Barbara et al (11) determinaron que el polen de la Apis mellifera fue eficiente en el control de microorganismos del tipo Gram positivo, mientras que para los Gram negativos su eficiencia era menor debido a factores propios de su estructura química a nivel de pared celular

En su estudio, en donde determinaron las características microbiológicas de polen de 3 géneros de abejas sin aguijón (Melipona, Scaptotrigona y Frieseomellita) Santa Barbara et al (19) determinaron que estas muestras no presentaron crecimiento de microorganismos tales como: Salmonella $s p$, esporas de Clostridium sulfito reductor, coliformes totales, Escherichia coli y Staphylococcus coagulasa positivos. Encontrando únicamente un patrón de crecimiento, en todas las muestras evaluadas, para los mesófilos aerobios. Estos resultados muestran la disparidad entre los resultados microbiológicos entre los géneros de abejas por lo que los manejos en cada uno de los sistemas productivos a nivel de transformación, debe adecuarse a este comportamiento.
Kačániová et al (30) determinaron la presencia de aflatoxinas producidas por hongos en muestras de polen congelado, a través de la técnica de ELISA, determinando que las micotoxinas ZON y T2 aparecieron en todas las muestras de polen, mostrando la gran importancia que tiene en salud publica la no presencia de estos microorganismos en los productos alimenticios, los cuales están presentes en polen SECO en tres apiarios evaluados apiarios evaluados.

Los procesos de conservación del polen (secado o congelado), según Estevinho et al (31) muestran influencias estadísticamente significativas con respecto a su composición fisicoquímica, junto con factores medio ambientales como la temporada de recolección y las especies botánicas presentes, y quizá esto podría influir en las condiciones microbiológicas debido a que, como se evidenció anteriormente, el polen tiene capacidad antimicrobiana que puede ser afectada por esta concentración de nutrientes. Los mismos autores, mencionan que es mejor consumir polen congelado a una temperatura de $-20^{\circ} \mathrm{C}$ que polen seco.

Los resultados del presente estudio muestran unas mejores características microbiológicas para el polen congelado en comparación con el polen seco para la mayoría de los microorganismos evaluados. Resulta necesario a partir de aquí evaluar también la calidad microbiológica de estos mismos procesos cuando el producto es sometido a diferentes tiempos de almacenamiento, y los posibles cambios en sus características organolépticas debido a este proceso.

\section{Conflicto de intereses}

Los autores declaramos que no tenemos conflicto de intereses.

\section{REFERENCIAS}

1. Álvarez A, Campos G. Diversidad y criterios microbiológicos en polen usado como suplemento alimenticio para humanos. Digital @UAQro. 2017; 10(2):218-229. https://www.uaq.mx/investigacion/revista ciencia@uaq/ArchivosPDF/v10-n2/art16 numpagina.pdf
2. Arruda V, Dos Santos A, Figuereido D, Silva E, Castro A, Fernandes M, Almeida L. Microbiological quality and physicochemical characterization of Brazilian bee pollen. J Apic Res. 2017; 56(3):231-238. http://dx.doi.or $\mathrm{g} / 10.1080 / 00218839.2017 .1307715$ 
3. Vargas JC. Canales y márgenes de comercialización de los productos apícolas en la provincia centro (departamento de Boyacá) [Tesis de Maestría]. Universidad Nacional de Colombia: Colombia; 2014. http://bdigital. unal.edu.co/45126/1/2577419.2014.pdf

4. Mauriello G, De Prisco A, Di Prisco G, La Storia A, Caprio E. Microbial characterization of bee pollen from the Vesuvius area collected by using three different traps. PLoS ONE. 2017; 12(9):e0183208. https://doi. org/10.1371/journal.pone.0183208

5. De-melo A, Estevinho $M$, Almeida L. A diagnosis of the microbiological quality of dehydrated bee-pollen produced in Brazil. Let Appl Micr. 2015; 61(5):477-483. https://doi.org/10.1111/lam.12480

6. Campos M, Bogdanov S, Bicudo L, Szczesna T, Mancebo Y, Frigeiro $C$ et al. Pollen composition and stadardisation of analytical methods. J Apicul Res. 2008; 47(4):156163. https://doi.org/10.1080/00218839.2 $\underline{008.11101443}$

7. Morais M, Moreira L, Feás X, Estevinho L. Honeybee-collected pollen from five Portuguese Natural Parks: Palynological origin, phenolic content, antioxidant properties and antimicrobial activity. Food Chem Toxicol. 2011; 49(5):1096-1101. https://doi.org/10.1016/j.fct.2011.01.020

8. Pascoal A, Rodrigues S, Texeira A, Feás X, Estevinho L. Biological activities of comercial bee pollens: Antimicrobial, antimutagenic, antioxidant and anti-inflammatory. Food Chem Toxicol. 2014; 63(1):233-239. https://doi.org/10.1016/j.fct.2013.11.010

9. Moita E, Sousa C, Andrade P, Fernandes $F$, Pinho P, Silva $L$ et al. Effects of Echium plantagineum $L$. bee pollen on basophil degranulation: Relationship with metabolic profile. Molecules. 2014; 19(7):1063510649. https://doi.org/10.3390/ $\underline{\text { molecules } 190710635}$

10. Komosinska-Vassev K, Olczyk P, Kafmierczak J, Mencner L, Olczyk K. Bee pollen: Chemical composition and therapeutic application. Evid Based Complement Altern. 2015; 1(1):1-6. http://dx.doi.org/10.1155/2015/297425
11. Santa Bárbara $M$, Moreira $M$, Machado C, Chambó E, Pascoal A, Carvalho C, Da Silva G, Delerue C, Estevinho L. Storage methods, phenolic composition, and bioactive properties of Apis mellifera and Trigona spinipes pollen. J Apic Res. 2020; 59(1)-125-134. https://doi.org/10.1080/0 $\underline{0218839.2019 .1708595}$

12. Graystock P, Yates K, Darvill B, Goulson D, Hughes W. Emerging dangers: deadly effects of an emergent parasite in a new pollinator host. J Inv Path. 2013; 114(2):114-119. https://doi.org/10.1016/j.jip.2013.06.005

13. Goulson D, Hughes W. Mitigating the anthropogenic spread of bee parasites to protect wild pollinators. Biol Cons. 2015; 191(1):10-19. http://dx.doi.org/10.1016/j. biocon.2015.06.023

14. Tarazona L, Villate J, Forero E, Grijalba J, Vargas J, Andrade R. Presencia de microorganismos micóticos en leche cruda de tanques de enfriamiento en el Altiplano Boyacense (Colombia). Rev CES Med Zootec. 2019; 14(2):8-17. http://dx.doi. org/10.21615/cesmvz.14.2.1

15. Hani B, Dalila B, Saliha D, Harzallah D, Ghadbane M, Khennouf S. Microbiological sanitary aspects of pollen. Adv Environ Biol. 2012; 6(4):1415-1420. http://www. aensiweb.com/old/aeb/2012/1415-1420.pdf

16. Nardoni S, D'Ascenzi C, Rocchigiani G, Moretti V, Mancianti F. Occurrence of molds from bee pollen in Central Italy - A preliminary study. Ann Agri Environ Med. 2016; 23(1):103-105. http://dx.doi. org/10.5604/12321966.1196862

17. Instituto de hidrología, meteorología y estudios ambientales [Internet]. Boletín meteorológico. 2019. [citado 11 septiembre de 2019]. Disponible en: http://www.ideam. gov.co/web/tiempo-y-clima/tiempo-clima

18. Belhadj H, Bouamra D, Dahamna S, Harzallah D, Ghadbane M, Khennouf S. Microbiological sanitary aspects of pollen. Adv Envir Biol. 2012; 6(4):1415-1420. http://www. aensiweb.com/old/aeb/2012/1415-1420.pdf 
19. Santa Barbara M, Machado C, Da Silva G, Lima F, Lopes C. Caracterizações microbiológica e físico-química de pólens armazenados por abelhas sem ferrão. Braz J Food Technol. 2018; 21(1):e2017180. https://doi.org/10.1590/1981-6723.18017

20. García D, Rojas M, Sánchez J. Contenido microbiológico cultivable de tracto intestinal y polen almacenado de Apis mellifera (Hymenoptera: Apidae). Acta Biol Col. 2006; 11(1):123-129. https://revistas. unal.edu.co/index.php/actabiol/article/ view/27150/27423

21. Ley 18284: Código Alimentario Argentino. Ministerio de salud y desarrollo social: Argentina; 1969. https://www.argentina. gob.ar/anmat/codigoalimentario

22. Norma oficial mexicana: NOM-092SSA1-1994. Secretaria de salud de México: México; 1994. http://www.salud.gob.mx/ unidades/cdi/nom/092ssa14.html

23. Ministério da agricultura e do abastecimento [Internet]. Regulamentos Técnicos de Identidade e Qualidade de Pólen Apícola. 2001. [citado 11 agosto de 2019]. https:// www.apacame.org.br/mensagemdoce/60/ normas.htm

24. De Melo A, Estevinho M, Sattler J, Souza, B, Freitas A, Barth M, Almeida L. Effect of processing conditions on characteristics of dehydrated bee-pollen and correlation between quality parameters. LWT-Food Sci Techn. 2016; 65(1):808-815. https://doi. org/10.1016/j.Iwt.2015.09.014

25. Estevinho L, Rodrigues $S$, Pereira A, Feás $X$. Portuguese bee pollen: palynological study, nutritional and microbiological evaluation. Int J Food Sci Techn. 2012; 47(1):429435. https://doi.org/10.1111/j.13652621.2011.02859.x
26. Nogueira C, Iglesias A, Feás X, Estevinho L. Commercial bee pollen with different geographical origins: A comprehensive approach. Int J Mol Sci. 2012; 13(9):1117311187 . https://doi.org/10.3390/ ijms130911173

27. Feás $X$, Vázquez $M$, Estevinho L, Seijas J, Iglesias A. Organic bee pollen: Botanical origin, nutritional value, bioactive compounds, antioxidant activity and microbiological quality. Molecules. 2012; 17(7):8359-8377. https://doi.org/10.3390/ molecules 17078359

28. Puig Y, Del risco C, Pazos V, Leiva V, García R. Comparación de la calidad microbiológica del polen apícola fresco y después de un proceso de secado. Rev CENIC Cien Biol. 2012; 43(1):23-27.

29. Santa Bárbara M, Santiago C, da Silva G, Dias L, Estevinho L, Lopes C. Microbiological assesment, nutritional characterization and phenolic compounds of bee pollen from Mellipona mandacaia Smith, 1983. Molecules. 2015; 20:12525-12544; http:// dx.doi.org/10.3390/molecules200712525

30. Kačániová M, Juráček M, Chlebo R, Kňazovická $V$, Kadasi-Horáková M, Kunová $S$, et al. Mycobiota and mycotoxins in bee pollen collected from different areas of Slovakia. J Envir Sci Hea, Part B: Pest, Food Cont, and Agr Was. 2012; 46(7):623-629. http:// dx.doi.org/10.1080/03601234.2011.589322

31. Estevinho L, Dias T, Anjos O. Influence of the Storage Conditions (Frozen vs. Dried) in Health-Related Lipid Indexes and Antioxidants of Bee Pollen. Eur J Lipid Sci Tecnol. 2019; 121(1):1800393. https://doi. org/10.1002/ejlt.201800393 